\title{
New trends on photopolymers
}

\author{
M. Ortuño ${ }^{* a}$, E. Fernández ${ }^{\mathrm{b}}, \mathrm{S}$. Gallego $^{\mathrm{a}}$, A. Márquez ${ }^{\mathrm{a}}, \mathrm{C} . \mathrm{Neipp}^{\mathrm{a}}$, I. Pascual ${ }^{\mathrm{b}}, \mathrm{A}$. Beléndez $^{\mathrm{a}}$ \\ ${ }^{a}$ Dpto. de Física, Ingeniería de Sistemas y Teoría de la Señal, Universidad de Alicante \\ ${ }^{\mathrm{b}}$ Dpto. de Óptica, Farmacología y Anatomía, Universidad de Alicante \\ *Apartado 99, E-03080 Alicante, Spain, mos@ua.es
}

\begin{abstract}
Photopolymers are light sensitive materials with adequate characteristics for application as holographic recording media in new devices as optical elements and holographic memories. A specific set of parameters are required from the photopolymers: good energetic sensitivity to save energy during the recording process, an adequate spectral sensitivity, a higher resolution to let the necessary fidelity in the stored information reproduction, a better environmental compatibility including low toxicity, easy making management and good recycling properties. We present the main research lines in this area and the last results obtained with the new photopolymeric materials that we are developing.
\end{abstract}

Keywords: Holography, sustainable photopolymer, green photopolymer, photopolymer with environmental properties, data storage holographic recording material

\section{INTRODUCTION}

Photopolymers that absorb in the visible spectrum are the subject of intense study since they make it possible to use commercial lasers emitting in this region of the spectrum. ${ }^{1}$ These materials are useful for different applications such as in the development of holographic memories, holographic optical elements or as holographic recording media. ${ }^{2}$ Usually, photopolymers have a photoinitiator system that absorbs light and generates free radicals that initiate the radical polymerization reaction of one or several different monomers. In the case of holographic recording, the basic mechanism of hologram formation involves modulation of the refractive index between polymerized and non-polymerized zones, corresponding to the "bright" and "dark" zones respectively, in the diffraction grating generated due to the interference of the recording beams.

There are many types of photopolymers that may be differentiated by the type of binder, since this component determines to a great extent the choice of monomer, dye and initiator used in the photopolymer. ${ }^{3}$ Examples of photopolymers with a hydrophobic binder are polyesters of methacrylic acid, which contain acrylic ester type monomers. Photopolymers with a hydrophilic binder include hybrid materials made by the sol-gel procedure. Photopolymers with a poly(vinyl alcohol) (PVA) or gelatin binder and monomers related to acrylamide (AA) are also hydrophilic. All these photopolymers have an undesirable feature, the toxicity of their components and their low environmental compatibility, particularly if we analyse the life cycle of the devices made with these materials and their interaction with the environment. The environmental compatibility and cycle of life are important features that must always be considered when developing new materials. ${ }^{4}$

Hydrophilic photopolymers with AA as the polymerizable monomer are versatile materials for use as holographic recording media. In layers up to $300 \mu \mathrm{m}$ thick they have been used to obtain holograms with a high diffraction efficiency and good energetic sensitivity. These photopolymers have good properties, in particular: their good energetic sensitivity compared with that of other available materials; the possibility of easily adapting their spectral sensitivity to the type of recording laser used by simple changing the sensitizer dye; high diffraction efficiency, together with an acceptable resolution and signal/noise ratio.

In this study we analyze the behaviour of an approximately $1 \mathrm{~mm}$ thick polyvinyl alcohol/acrylamide photopolymer with a view to its possible use in WORM (write once read many) type holographic memories. Using a standard holographic set-up, we recorded unslanted diffraction gratings in the material and determined the response of the photopolymer in

Practical Holography XXII: Materials and Applications, edited by Hans I. Bjelkhagen, Raymond K. Kostuk, Proc. of SPIE Vol. 6912, 691207, (2008) · 0277-786X/08/\$18 · doi: 10.1117/12.762658 
terms of the different holographic parameters: diffraction efficiency, sensitivity and angular response. In particular, we analyzed the peculiarities of this type of photopolymer when very thick layers are used.

Recently, their good qualities have been demonstrated in the development of devices with industrial applications such as holographic recording materials or holographic memories. ${ }^{5,6}$ In this respect, our research team has developed a photopolymer in layers $1 \mathrm{~mm}$ thick for use as a holographic memory, and its main holographic characteristics have been evaluated. $^{7-9}$

The main drawback of an AA-based photopolymer as far as the environment is concerned is the acrylamide, a substance which has been known to be carcinogenic for many years. Recent investigations confirm the toxic potential of AA. ${ }^{10-11}$

In order to develop a new photopolymer with environmental compatibility we used as reference the AA-based photopolymer in layers up to $1 \mathrm{~mm}$ thick, and evaluated the most environmentally unfriendly components in order to propose an alternative. We developed a new photopolymer with photochemical and holographic features similar to those of the standard material but with an improved design from the environmental point of view. We have applied for a patent for this new material. ${ }^{12}$

\section{EXPERIMENTAL}

\subsection{Preparation of the material}

In recording materials based on PVA as binder, a solution of PVA in water forms the matrix and this is used to prepare the mixture of monomer and photopolymerization initiator system. The mixture is usually deposited in a layer on a glass plate and after evaporation of part of the water, a solid plastic film is formed which constitutes the holographic recording material. The conventional method of depositing the layer consists in using a manual or automatic depositor, which by means of a rod or calibrated opening deposits the solution in a uniformly thick layer on a glass plate. In order to increase the final thickness of the layer, the PVA concentration in the initial solution is increased, since in this way a greater amount of water is retained by the PVA in the dry layer. However, this also means that there is a significant increase in the viscosity of the solution. Since there is a limit to the viscosity of the solutions that can be used with the depositors, and bearing in mind the process of preparation and subsequent cutting of the coated plates, the maximum thickness that can be obtained by this method is between 200 and $300 \mu \mathrm{m}$. The use of molds, depositing the photopolymer solution by gravity is another method of preparing the layers which enables greater thicknesses to be obtained. In this method we prepare the solutions using a conventional magnetic stirrer, under red light and in standard laboratory conditions (temperature, pressure, relative humidity). If the viscosity is too high to use a magnetic stirrer, we stir slowly by hand to prevent the formation of air bubbles, followed by the application of a vacuum to both the prepared solution and the coated mold so that any eventual air bubbles are completely eliminated. ${ }^{7}$ The solutions are deposited by gravity, in polystyrene or poly(methylmethacrylate) molds, and left in the dark to allow the water to evaporate, while recording the laboratory conditions (temperature and relative humidity, RH) during the process. When part of the water has evaporated, the dry material is removed from the mold, cut into squares and adhered, without the need for adhesive, to the surface of glass plates measuring $6.5 \times 6.5 \mathrm{~cm}$. The plates are then ready for exposure, which takes place immediately. The thickness of the material is measured using a conventional micrometer.

Photopolymer A: The optimized composition of PVA/AA prepolymer solution for 40-300 $\mu$ m layers can be seen in Table 1. 
Table. 1. Optimized composition of photopolymer A solution for 40-300 $\mu \mathrm{m}$ layers and parameters to be considered in its preparation.

\begin{tabular}{|l|l|}
\hline AA & $0.44 \mathrm{M}$ \\
\hline Triethanolamine (TEA) & $0.2 \mathrm{M}$ \\
\hline yellowish eosin (YE) & $2.44 \times 10^{-4} \mathrm{M}$ \\
\hline PVA (Mw $=27000$ uma) & $13.30 \%$ w/v \\
\hline Drying time & $\begin{array}{l}\text { Variable depending on the relative } \\
\text { humidity. RH } 45 \%, 2 \text { days }\end{array}$ \\
\hline
\end{tabular}

Photopolymer A: The optimized composition of PVA/AA prepolymer solution for up to $1 \mathrm{~mm}$ layers can be seen in Table 2.

Table. 2. Optimized composition of photopolymer A solution for up to $1 \mathrm{~mm}$ layers and parameters to be considered in its preparation.

\begin{tabular}{|l|l|}
\hline AA & $0.34 \mathrm{M}$ \\
\hline TEA & $0.15 \mathrm{M}$ \\
\hline YE & $12.0 \times 10^{-5} \mathrm{M}$ \\
\hline PVA $(\mathrm{Mw}=130000 \mathrm{uma})$ & $13.30 \% \mathrm{w} / \mathrm{v}$ \\
\hline Drying time & $\begin{array}{l}\text { Variable depending on the relative } \\
\text { humidity. RH } 45 \%, 6 \text { days }\end{array}$ \\
\hline
\end{tabular}

\subsection{Environmental characteristics of the standard AA-based photopolymer}

Standard photopolymer (Tables 1,2) is composed of AA as polymerizable monomer, triethanolamine as coinitiator and plasticizer, yellowish eosin as dye, PVA as binder and a small proportion of water as additional plasticizer. It may also contain N,N'-methylene-bis-acrylamide (BMA) as crosslinking monomer.

This material has a hydrophilic binder and this implies that during its production the main solvent used is water. In fact, our photopolymer does not use any additional cosolvent. Any hypothetical products made of this photopolymer could also be eliminated, once their useful life was over, by dissolving in water. Therefore, this material has an advantage over hydrophobic photopolymers because it avoids the use of petroleum based solvents which are toxic and flammable.

The problem is their composition. Of the different components, AA and BMA are toxic monomers, the former being more toxic than the latter. YE also poses problems due to the $4 \mathrm{Br}$ atoms in its molecule. ${ }^{13}$ The decomposition byproducts of YE are not photoactive but they can interact with the environment to generate halogenated substances which are toxic. All dyes derived from fluorescein have these characteristics, such as Bengal rose or B erytrosin dyes, commonly used in hydrophilic photopolymers with AA as monomer. As evidence of their toxicity, we can mention the research being done on their possible application as pesticides. ${ }^{14}$

Bearing in mind these special features, it is necessary to find a new monomer and dye with a low potential toxicity that may replace AA and YE in the development of a new biocompatible photopolymer. The new monomer and dye must be compatible with the hydrophilic binder and the photopolymer must be able to obtain comparable results.

\subsection{Dye substitution}


The dyes for the new photopolymer must have specific properties to make the system feasible. It is not possible to use commercial fluorescein based substances because they all contain halogen atoms in their molecules. The dye must be water soluble and have no halogen atoms or functional groups that could make the molecule toxic. It must absorb at visible wavelengths and to have an adequate efficiency in radical free generation to let a radical chain polymerization, either by radicals from the dye molecule itself or by those from TEA through a redox reaction, as happens in the case of the YE/TEA pair.

It is known that the riboflavin molecule (component of the B2 vitaminic complex) and flavin derivatives in general absorb in the $500 \mathrm{~nm}$ region. These substances are photosensitizers because they have a high intersystem crossing quantum yield. Upon light absorption they reach the triplet excited state and react with electron donors to generate radical intermediates.

In the new photopolymer we use the sodium salt 5'-riboflavin monophosphate (PRF) as dye, bearing in mind that this substance is water soluble and exists in the environment, so it is not likely to cause environmental problems.

$900 \mu \mathrm{m}$ thick layers of photopolymer B are made with the composition shown in Table 3 . It can be seen that this photopolymer does not contain YE as opposed to the standard photopolymer A. In Figure 1 the transmission spectrums for photopolymers A and B can be seen. For photopolymer A the highest absorption takes place in the 485-550 nm region, whereas for photopolymer $\mathrm{B}$, with $\mathrm{PRF}$, the main absorption is at wavelengths lower than $500 \mathrm{~nm}$. The argon laser used in the hologram recording experiments is tuned at $514 \mathrm{~nm}$ where the absorption for photopolymer $\mathrm{A}$ is higher than that for photopolymer $\mathrm{B}, \mathrm{T}=0.4 \%$ and $\mathrm{T}=46.7 \%$ respectively.

Table. 3. Composition of the prepolymer solution in molarity, PVA in percentage.

\begin{tabular}{|l|c|c|c|c|}
\hline & $\mathrm{B}$ & $\mathrm{C}$ & $\mathrm{D}$ & $\mathrm{E}$ \\
\hline $\mathrm{AA}$ & 0.34 & - & - & - \\
\hline NaAO & - & 0.31 & 0.34 & 0.34 \\
\hline YE & - & $9.0 \times 10^{-5}$ & - & - \\
\hline PRF & $1.0 \times 10^{-3}$ & - & $1.0 \times 10^{-3}$ & $1.0 \times 10^{-3}$ \\
\hline TEA & 0.15 & 0.15 & 0.15 & 0.15 \\
\hline PVA & $13.4 \%$ & $13.4 \%$ & $13.4 \%$ & $13.4 \%$ \\
\hline DHEBA & - & - & - & $6.4 \times 10^{-3}$ \\
\hline
\end{tabular}

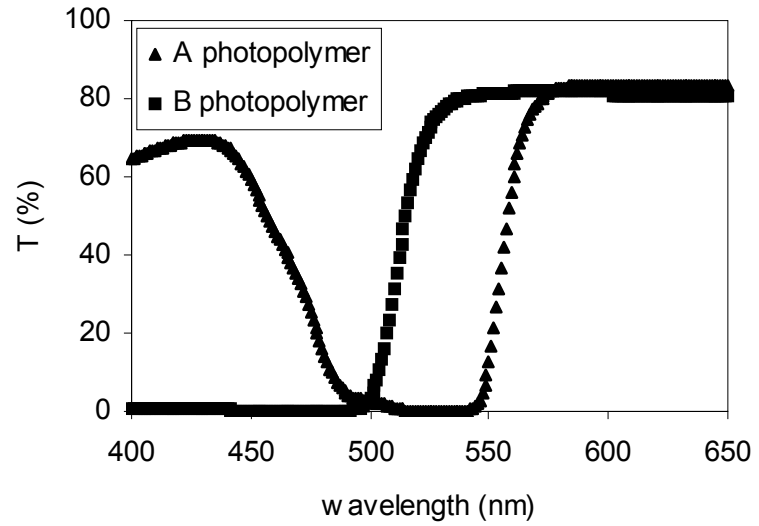

Fig. 1. Transmission spectrums for photopolymers A and B. 


\subsection{Monomer substitution}

For the monomer substitution it is necessary to use another vinyl monomer that is less toxic than AA. The monomer must be water soluble, it must not evaporate during the preparation of layers and it has to react through a radical chain mechanism. Initially, we used acrylic acid as monomer but it evaporates with water during the process of drying of the layers so the concentration in the photopolymer is very low and the performance of the material is poor. Therefore, we converted the acrylic acid into sodium acrylate by using sodium hydroxide in the initial solution used to prepare the layers. The toxicity of sodium acrylate is lower than that of AA. ${ }^{15}$ In Table 3 can be seen the composition of C photopolymer, in which AA is substituted by sodium acrylate (NaAO).

\subsection{Simultaneous monomer and dye replacement}

In photopolymer D we replaced AA by NaAO and YE by PRF.

\subsection{Crosslinking}

N,N'-Methylenebisacrylamide (BMA), a known reproductive toxicant, is the crosslinker usually used in the standard photopolymer with AA and YE. In this study we propose N,N'-(1,2-dihydroxyethylene)bisacrylamide (DHEBA) as an alternative. DHEBA has occasionally been used in hydrophilic photopolymers due to its good solubility in water. This molecule is suitable for the new photopolymer because its two hydroxyl groups are compatible with the structure of the sodium polyacrylate generated in the photopolymerization. In this manner, hydrogen bonds may be formed with the PVA binder, and TEA and water plasticizers. Although there are no studies in the bibliography that suggest this substance is toxic, a future research could prove a certain toxicity level, but minor than BMA. Photopolymer E has NaAO, PRF and DHEBA.

\subsection{Preparation of the biophotopolymer}

The solutions, which composition can be seen in Table 3, with water as solvent, are deposited, using the force of gravity, in polystyrene molds, and left in the dark $\left(\mathrm{RH}=40-45 \%, \mathrm{~T}=20-23^{\circ} \mathrm{C}\right)$. When part of the water has evaporated (about 6 days), the layer has enough mechanical resistance and it can be extracted from mold without deformation. The solid film is cut into squares $900 \mu \mathrm{m}$ thick and adhered, without the need for adhesive, to the surface of glass plates measuring $6.5 \times 6.5 \mathrm{~cm}$. The plates are then ready for exposure, which takes place immediately. ${ }^{16}$

\subsection{Holographic setup}

To study the behavior of these photopolymers as a holographic recording material, we obtained diffraction gratings using a holographic setup. The experimental device is shown in Figure 2. An Argon laser at a wavelength of $514 \mathrm{~nm}$ was used to store diffraction gratings by means of continuous laser exposure. The laser beam was split into two secondary beams with an intensity ratio of $1: 1$. The diameters of these beams were increased to $1.5 \mathrm{~cm}$ with an expander, while spatial filtering was ensured. The object and reference beams were recombined at the sample at an angle of $16.8^{\circ}$ to the normal with an appropriate set of mirrors, and the spatial frequency obtained was 1125 lines $/ \mathrm{mm}$. The working intensity at 514 $\mathrm{nm}$ was $5 \mathrm{~mW} / \mathrm{cm}^{2}$. The diffracted and transmitted intensity were monitored in real time with a He-Ne laser positioned at Bragg's angle $\left(20.8^{\circ}\right)$ tuned to $633 \mathrm{~nm}$, where the material does not polymerize. In order to obtain transmission and diffraction efficiency as a function of the angle at reconstruction we placed the plates on a rotating stage. The transmission and diffraction efficiency (TE and DE respectively) were calculated as the ratio of the transmitted and diffracted beam, respectively, to the incident power. 


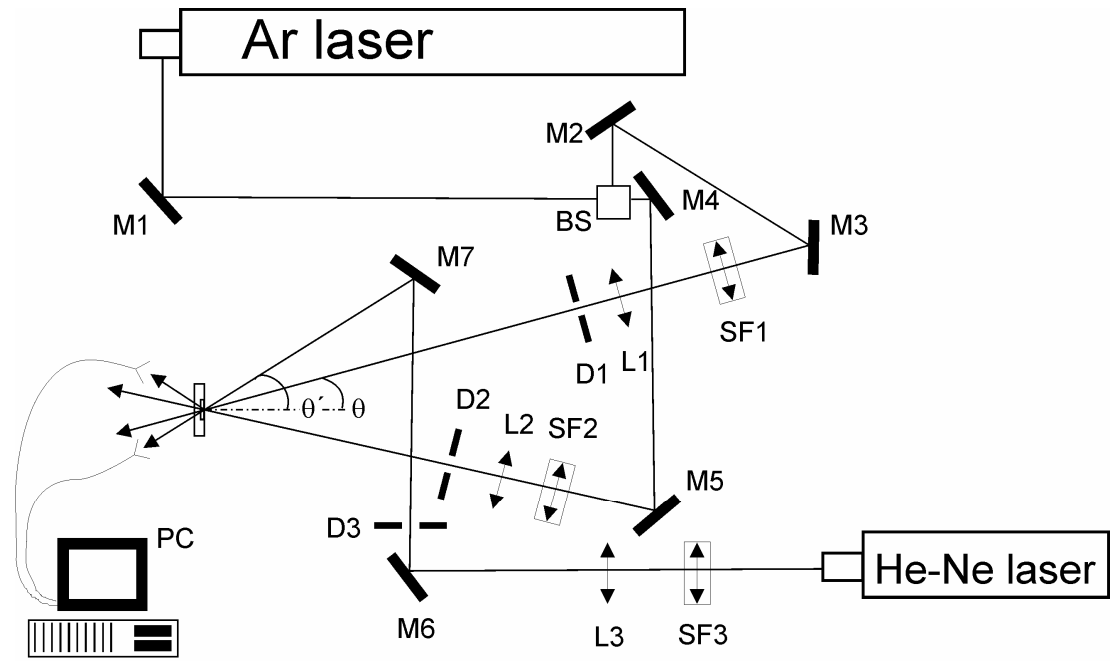

Fig. 2. BS: Beamsplitter, Mi: mirror, SFi: spatial filter, Li: lens, Di: diaphragm, PC: data recorder.

\section{RESULTS}

In Figure 3 we can see the diffraction efficiency versus exposure energy in the recording of the diffraction grating recorded in photopolymer A, $80 \mu \mathrm{m}$ thick layer. The maximum diffraction efficiency (DEmax) is higher than $77 \%$ with an energetic sensitivity about $400 \mathrm{~mJ} / \mathrm{cm}^{2}$.

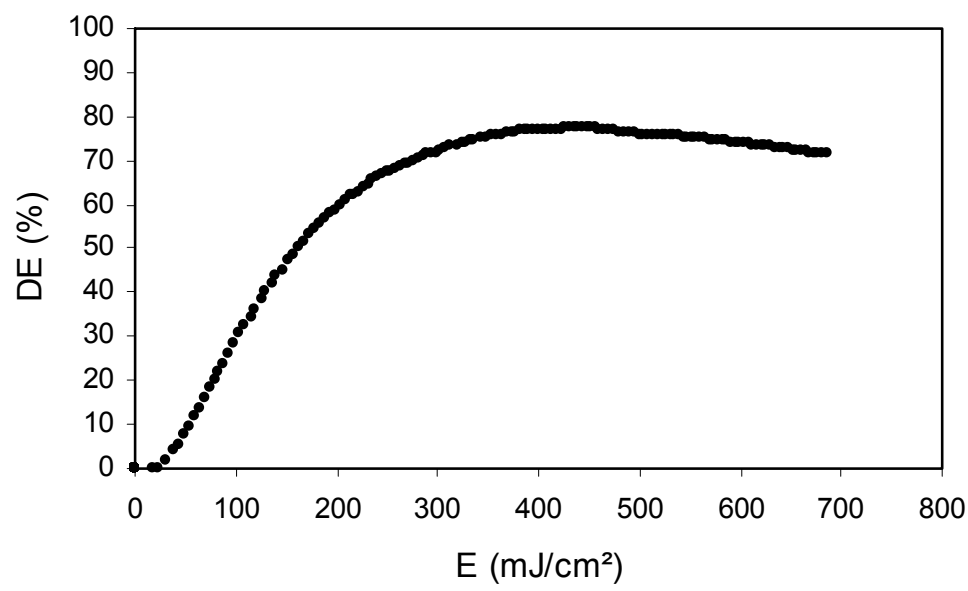

Fig. 3. Diffraction efficiency versus energy during recording for photopolymer A, $80 \mu \mathrm{m}$ thick layer.

In Figure 4 we can see the angular scan in the reconstruction of the hologram recorded in photopolymer A, $1 \mathrm{~mm}$ thick layer. The DEmax is higher than $75 \%$ with a small angular interval, about 0.4 degrees. The energetic sensitivity is 124 $\mathrm{mJ} / \mathrm{cm}^{2}$ 


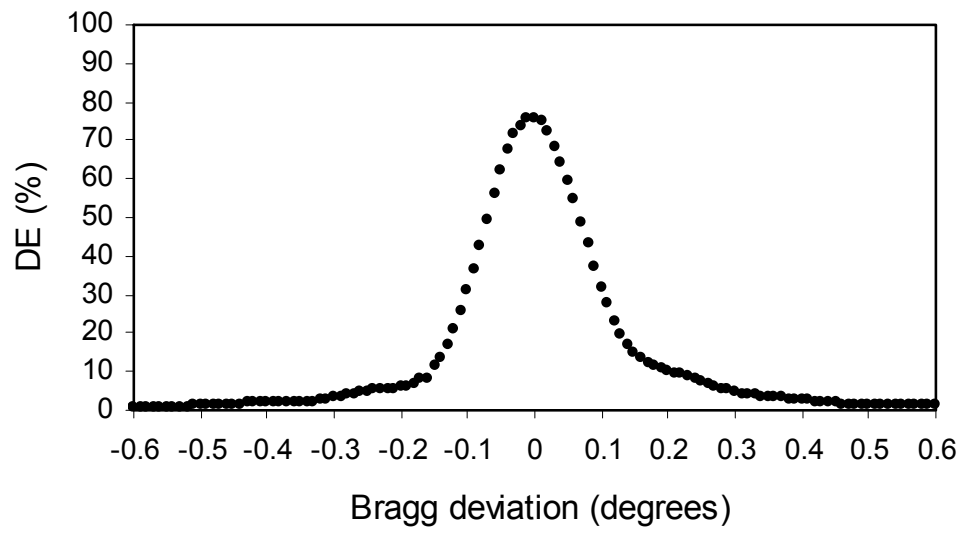

Fig. 4. Hologram reconstruction, photopolymer A, $1 \mathrm{~mm}$ thick layer.

In Figure 5 we can see the recording process of the holograms for B, C, D and E photopolymers. For photopolymer $\mathrm{C}$ the energy for DEmax is $\mathrm{S}_{\mathrm{C}}=39 \mathrm{~mJ} / \mathrm{cm}^{2}$, so replacing AA by NaAO has no influence on the diffraction characteristics of the polymer chains. For photopolymer A the polymer chains of the grating are made of polyacrylamide, and for photopolymer $\mathrm{C}$ of poly(sodium acrylate). In this experiment we obtained better energetic sensitivity with the NaAO photopolymer than with the standard photopolymer A (Figures 3,4), although the energetic sensitivity varies significantly with the water content in the photopolymer layer, which is in equilibrium with the environmental humidity.

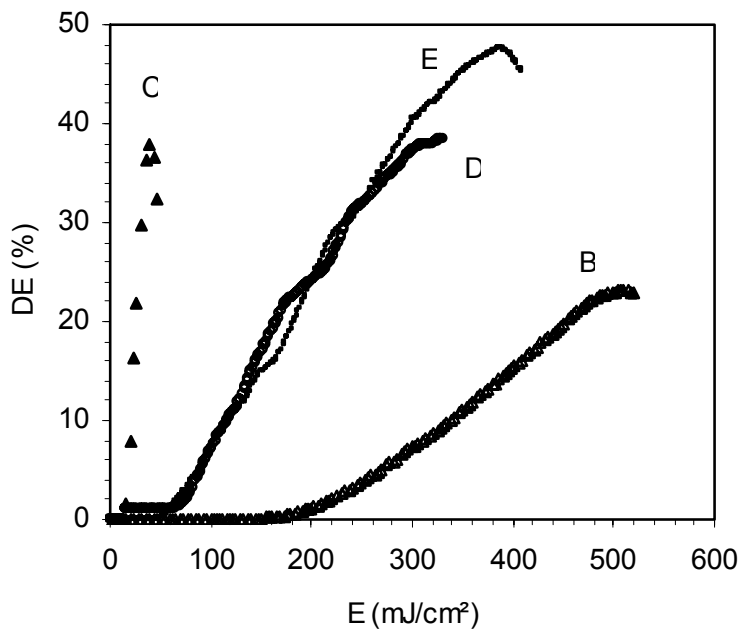

Fig. 5. Diffraction efficiency versus energy during recording for photopolymers B-E.

The other photopolymers need a considerably higher energy to reach the maximum diffraction efficiency $\mathrm{S}_{\mathrm{B}}=511$ $\mathrm{mJ} / \mathrm{cm}^{2}, \mathrm{~S}_{\mathrm{D}}=333 \mathrm{~mJ} / \mathrm{cm}^{2}, \mathrm{~S}_{\mathrm{E}}=338 \mathrm{~mJ} / \mathrm{cm}^{2}$, so this suggests that the YE/TEA redox pair initiation system has a better energetic sensitivity than PRF or the PRF/TEA combination.

Figure 6 shows the angular scan obtained after recording the holograms stored in the photopolymers. First, we can see the difference in the angular width of the curves for the YE/TEA photopolymers (Figure 4) and PRF photopolymers. So for photopolymer C DEmax is reached in an angular interval higher than $0.3^{\circ}$. On the other hand, for photopolymers $\mathrm{D}$ and $\mathrm{E}$, the angular interval is lower than $0.2^{\circ}$. This indicates a deeper polymer grating according to Kogelnik's theory. ${ }^{17}$ 


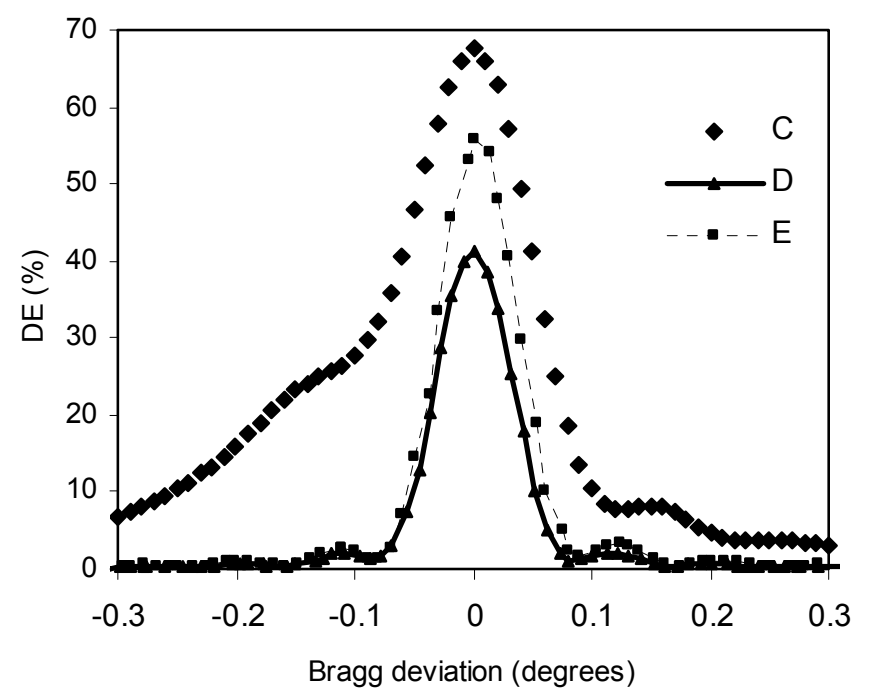

Fig. 6. Hologram reconstruction, photopolymers C-E.

This result is due to the lower absorption of PRF compared with YE for the recording wavelength and this implies that the recording laser beam penetrates the photopolymer thickness more easily. A small angular interval is very important when recording many holograms by multiplexing since this makes it more difficult for holograms to overlap, thereby allowing these holograms to be reconstructed more easily. In addition, more efficient use is made of the photopolymer thickness because the polymer grating is deeper in proportion to the decrease in the width of the angular response curve. ${ }^{18}$

Another important result is the high DEmax obtained (41-68\%). If we compare the values for photopolymers A (Figure 4) and $\mathrm{C}, 75 \%$ and $68 \%$ respectively, we can see that substitution of AA by NaAO does not introduce limitations on the performance of the stored diffraction grating.

We obtain a good result with the substitution of YE and AA by PRF and NaAO, DHEBA respectively: photopolymer E, DEmax $=56 \%$.

Photopolymer D has a DEmax $=41 \%$, which is lower than expected. However, it should be borne in mind that these photopolymers are exposed to variations in environmental conditions and, in particular, that the humidity is not controlled in these experiments.

\section{CONCLUSION}

We developed AA-based photopolymers in layers up to $1 \mathrm{~mm}$ thick with higher diffraction efficiency and good sensitivity that can be used to make holographic memories and holographic optical elements. We also developed a new photopolymer with better environmental compatibility and with similar holographic properties. The highly toxic components of the standard photopolymer were eliminated, thereby obtaining a material with a lower potential toxicity.

The new photopolymer has a small angular interval for the angular response curve $\left(0.2^{\circ}\right)$ that may be useful for the recording of many holograms by multiplexing. On the other hand, the byproducts derived from PRF after photodecomposition are also dyed and can initiate new polymerization reactions with a similar efficiency to that of the initial PRF molecule. Therefore, the dye is not a limiting factor, especially when many holograms are recorded. This photopolymer is an alternative to the traditional AA-based photopolymers that can be used as holographic recording material in holographic optical elements and data storage applications. 


\section{ACKNOWLEDGMENTS}

This work was supported by Ministerio de Educación y Ciencia, Spain (FIS2005-05881-C02-01, FIS2005-05881-C0202, АCOMP06-007, АCOMP07-020).

\section{REFERENCES}

1. R. A.Lessard, G. Manivannan, Selected Papers on Photopolymers: Physics, Chemistry and Applications, Proc. SPIE MS 114 (1995).

2. H. J. Coufal, D. Psaltis, Holographic Data Storage, G. T. Sincerbox, Springer-Verlag, New York 2000.

3. R. A. Lessard, G. Manivannan, Holographic Recording Materials: An Overview, in Holographic Materials, Proc. SPIE 2405, 2-23 (1995).

4. F. Pellaschiar, "Reduction of the utilization of VOC and their atmospheric emissions. Development of legislation and research in the use of less toxic solvents and reduced environmental impact," Eur. Coat. J. 81, 22-28 (2005).

5. E. Fernández, C. García, I. Pascual, M. Ortuño, S. Gallego, and A. Beléndez, "Optimization of a thick polyvinyl alcohol-acrylamide photopolymer for data storage using a combination of angular and peristrophic holographic multiplexing," Appl. Opt. 45, 7661-7666 (2006).

6. A. Márquez, C. Neipp, A. Beléndez, S. Gallego, M. Ortuño, and I. Pascual, "Edge-enhanced imaging with polyvinyl alcohol/acrylamide photopolymer gratings," Opt. Lett. 28, 1510-1512 (2003).

7. M. Ortuño, S. Gallego, C. García, C. Neipp, A. Beléndez, and I. Pascual, "Optimization of a $1 \mathrm{~mm}$ thick PVA/acrylamide recording material to obtain holographic memories: method of preparation and holographic properties," Appl. Phys. B 76, 851-857 (2003).

8. M. Ortuño, S. Gallego, C. García, C. Neipp, and I. Pascual, "Holographic characteristics of a $1 \mathrm{~mm}$ thick photopolymer to be used in holographic memories," Appl. Opt. 42, 7008-7012 (2003).

9. M. Ortuño, S. Gallego, C. García, I. Pascual, C. Neipp, and A. Beléndez, "Holographic characteristics of an acrylamide/bisacrylamide photopolymer in 40-1000 $\mu \mathrm{m}$ thick layers," Phys. Scr. T118, 66-68 (2005).

10. K. Hashimoto, and W. N. Aldridge, "Biochemical studies on acrylamide, a neurotoxic agent," Biochem. Pharmacol. 19, 2591-2604 (1970).

11. F. Mendel, "Chemistry, biochemistry, and safety of acrylamide. A review," J. Agric. Food. Chem. 51, 4504-4526 (2003).

12. M. Ortuño, S. Gallego, C. Neipp, A. Márquez, A. Beléndez, and I. Pascual, "Material de registro holográfico medioambientalmente compatible basado en acrilatos metálicos," Patent pending ES 200503113 (2005).

13. A. L. Lipman, Safety of xanthene dyes according to the U.S. Food and Drug Administration, ACS Symposium Series, American Chemical Society, USA, [Light-Activated Pest Control 616, $34-53$ (1995)].

14. J. R. Heitz, Insectic Mode Action, Academic (New York, USA, 1982).

15. J. M. Barnes, "Effects on rats of compounds related to acrylamide," British Journal of Industrial Medicine 1970.

16. M. Ortuño, E. Fernández, S. Gallego, A. Beléndez, and I. Pascual, "New photopolymer holographic recording material with sustainable design," Opt. Express 15(19), 12425-12435 (2007).

17. H. Kogelnik, "Coupled Wave Theory for Thick Hologram Gratings," Bell Syst. Tech. J. 48, 2909-2912 (1969).

18. S. Gallego, M. Ortuño, C. Neipp, A. Márquez, A. Beléndez, I. Pascual, J. V. Kelly, and J. T. Sheridan, "Physical and effective optical thickness of holographic diffraction gratings recorded in photopolymers," Opt. Express 13, 1939-1947 (2005). 